\title{
Cleansing and Care of a Deep and Narrow Wound with Easily Accessible Medical Equipment
}

\author{
Chan Min Chung, Seung Wan Tak, Hyoseob Lim, Sang Hun Cho \\ Department of Plastic and Reconstructive Surgery, Hallym University Sacred Heart Hospital, Hallym University College of Medicine, Anyang, Korea
}

\begin{abstract}
Of the many facets of managing wounds, mechanical dressing is one of the major concerns. A narrow and deep wound is particularly difficult to mechanically dress, because the small opening to the wound prevents effective gauze-wiping and irrigating with standard syringes. Here we present a case in which a 33-year-old patient with a narrow and deep perineal wound was provided mechanical cleansing with rectal tubes and Foley catheters. Irrigation with the tubes and catheters kept the deepest recesses of the wound clean for 6 months as the patient waited for his fractured pelvic bone to stabilize before he could undertake surgery to cover the wound. Underscoring the importance of effective cleansing in wound care, this case offers insights on new devices and methods.
\end{abstract}

Keywords: Perineum; Wound healing; Wound infection; Therapeutic irrigation

\section{Introduction}

A deep and narrow wound is hard to manage; mechanical cleansing beyond the edges of the opening and draining discharge are particularly challenging. Because these wounds have small openings, it is difficult to clean the wound bed with curettage or gauze-wiping. Physicians have attempted a variety of procedures to surmount this challenge, including continued irrigation and drainage, pulsed lavage cleansing and modifications thereof, irrigating with closed drain tubes, and the conventional practice of frequently changing dressings using gauze to drain capillaries [1-4].

This case presents a deep and narrow wound irrigated with rectal tubes and Foley catheters. Using easily available tools helped the irrigation and resulted in successful long-term wound care. The study was approved by the Institutional Review Board of Hallym Sacred Heart Hospital (IRB No. 2019-06-021-001) and performed in accordance with the principles of the Declaration of Helsinki. Written informed consent was obtained.

\section{Case}

A 33-year-old male patient sustained multiple traumatic injuries from a highway car accident. He suffered a wound on the left side of his perineum opening out towards the back, which would not drain naturally. Irrigation and drainage were performed five times every day. After each treatment, the physicians placed on the wound a piece of povidone-iodine-soaked gauze to drain the discharge through capillary action. The pus drainage almost completely subsided after 1 week. However, during follow-up a $17 \times 2 \times 11-\mathrm{cm}$ sized fluid collection was detected by computed tomography in an intramuscular area of the left gluteus maximus muscle. The fluid collection was com-

\author{
(D)

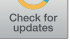 \\ Case Report \\ Received: July 27, 2019 \\ Revised: August 19, 2019 \\ Accepted: August 28, 2019 \\ Corresponding author: \\ Hyoseob Lim, M.D., Ph.D. \\ Department of Plastic and Reconstructive \\ Surgery, Hallym University Sacred Heart \\ Hospital, Hallym University College of \\ Medicine, 22 Gwanpyeong-ro 170beon-gil, \\ Dongan-gu, Anyang 14068, Korea \\ Tel: +82-31-380-3781 \\ Fax: +82-31-380-5980 \\ E-mail: hyoseob.lim@gmail.com
}

This is an Open Access article distributed under the terms of the Creative Commons Attribution Non-Commercial License (http://creativecommons.org/licenses/by-nc/4.0/) which permits unrestricted non-commercial use, distribution, and reproduction in any medium, provided the original work is properly cited.

(c) 2019 Korean Wound Management Society 

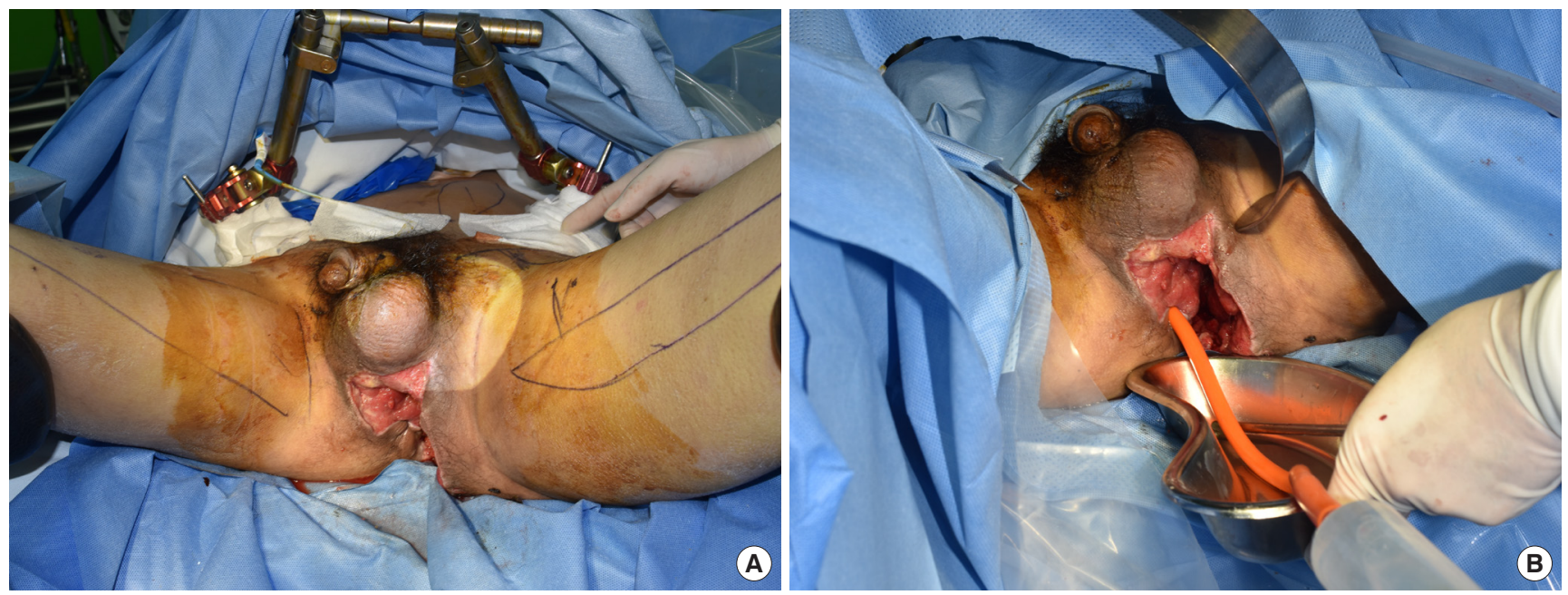

Fig. 1. Perineal wound 3 weeks after trauma. (A) The wound has an opening on the perineum of the patient. The wound involved the anus and a loop colostomy was performed so the wound was separated from the fecal contamination. (B) A rectal tube was inserted from the opening of the perineum. The irrigation was performed with the pressure directed by the physician and the leakage from the wound was collected in the tray placed underneath.
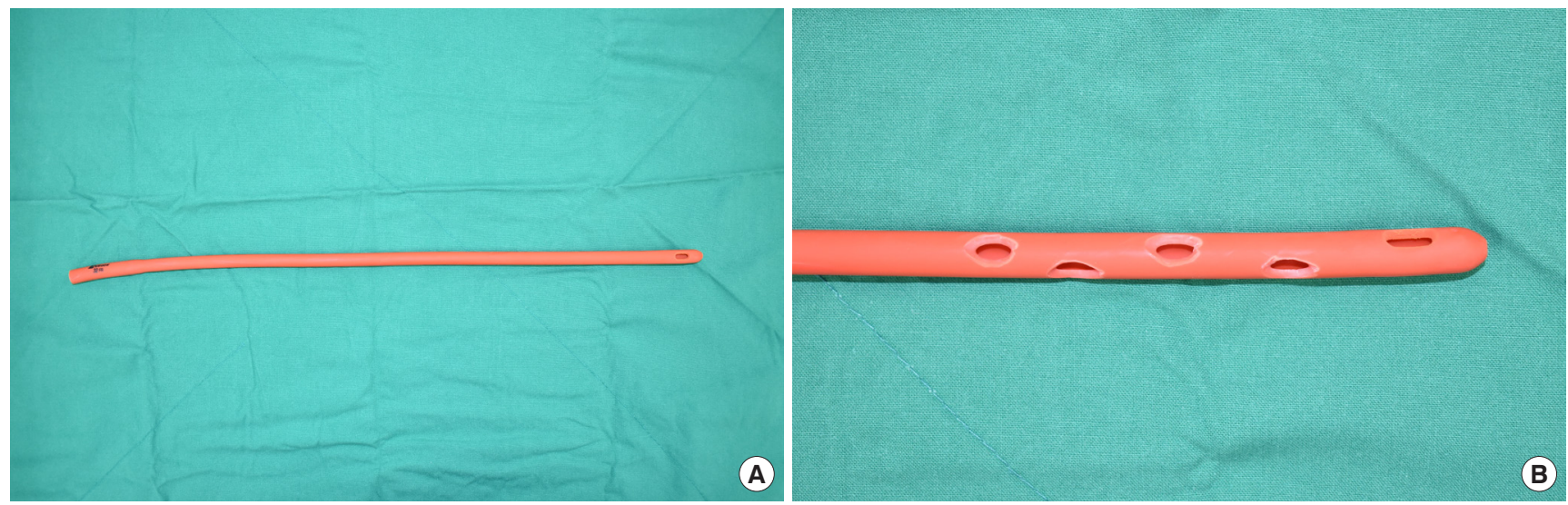

Fig. 2. Gross photography of the irrigation (rectal) tube. (A) The 32-Fr rectal tube shown is used for irrigation. (B) Distal part of the same rectal tube with several holes made.

municating with the deep perineal wound, deepening the wound.

The wound was in the shape of a deep tunnel with a dead end where pus collected. The pus would not drain naturally because the patient had fractured his pelvic bone and was unable to change his position. To drain the wound, a rectal tube was inserted all the way through the tunnel and saline irrigation was performed twice every day (Fig. 1). Normally, a rectal tube has only one opening at the end of the tube, so several holes were made to help irrigate the tunnel (Fig. 2). The tube was changed every 3 days, and was kept in position after the irrigation so as to facilitate even minimal natural drainage.

After a week, the fluid collection had decreased to $10 \times 3 \times 6$ $\mathrm{cm}$, and follow-up computed tomography confirmed that the rectal irrigation tube was positioned properly. Tube irrigation was continued until the pelvic bone was sufficiently stabilized after several orthopedic surgeries; the orthopedic department recommended postponing soft tissue reconstruction until the pelvic bone was stable enough. A 32-Fr rectal tube was used initially, but as the wound gradually narrowed it was irrigated with increasingly smaller tubes until it was replaced with a 14Fr Foley catheter (Fig. 3). The patient was given a perineal artery perforator flap surgery after his pelvic bone was stabilized 7 months after the initial trauma (Fig. 4). During the operation, the surgeon observed that the inside of the wound was clean and filled with granulation tissues owing to appropriate 


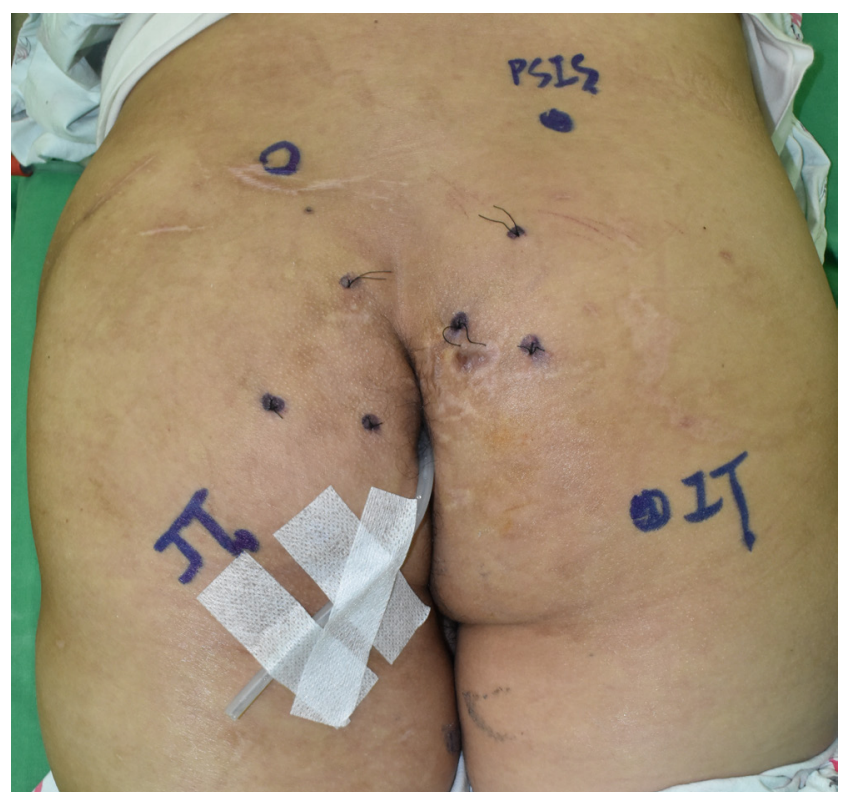

Fig. 3. Gross photograph of inserted Foley catheter. The tube was not removed after dressing, allowing the tube to facilitate natural drainage from the wound.

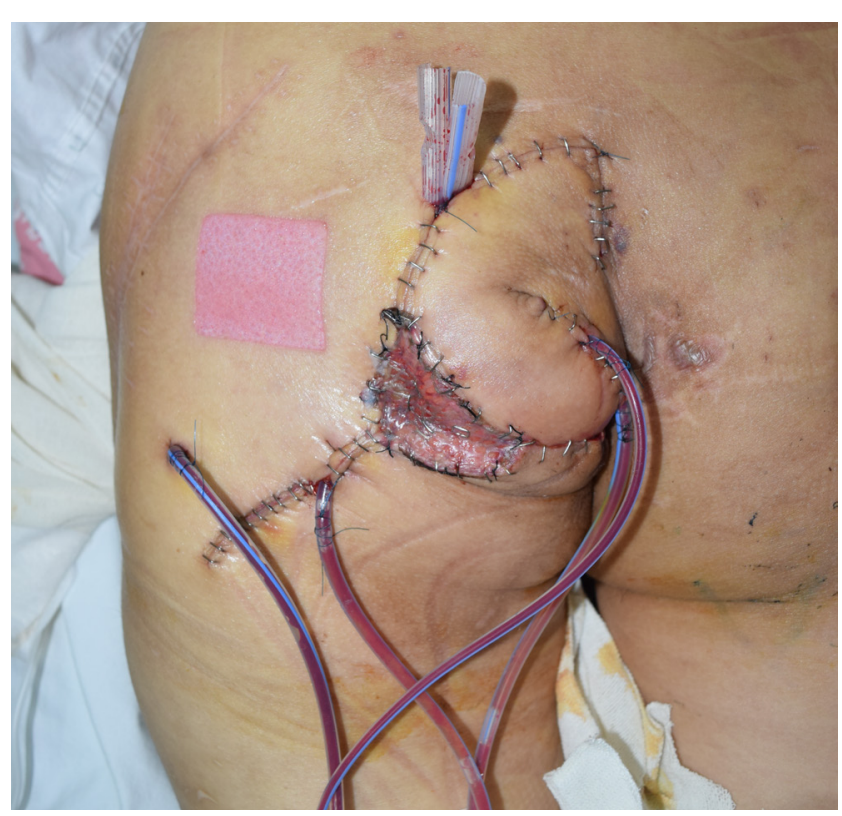

Fig. 4. Gross photograph after flap coverage. A perforator perineal artery flap was used to cover the soft-tissue defect after the pelvic bone was stabilized.

management. Three weeks after the flap coverage, the wound was almost fully healed with a minimal skin defect and without any complications such as trapped pus or fluid collection within. At 8 months after surgery, the wound exhibited no complications or recurring infections (Fig. 5).

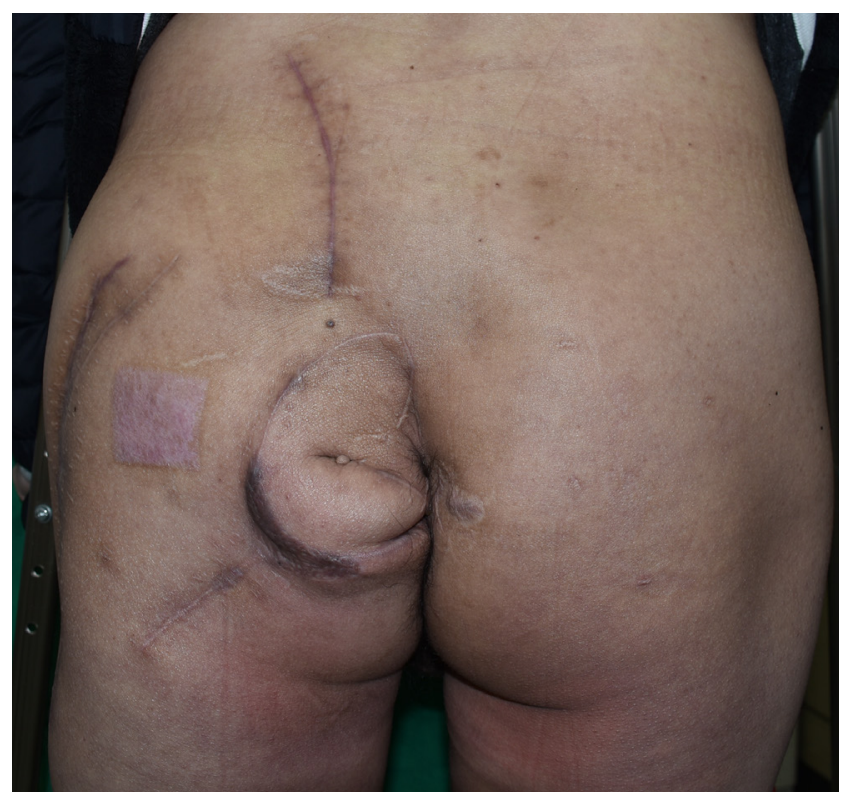

Fig. 5. Gross photography 8 months after flap coverage. Complete wound healing without any complications.

\section{Discussion}

The case presented a wound difficult to manage up to the point that soft tissue coverage was restored. Though it was precise and methodical irrigation that delivered successful outcomes, other wound treatments had also been attempted. Negative pressure wound therapy (NPWT) was tried several times, but it failed to drain the deeper part of the wound as effectively as the gauze soaking. Curettage and dressing with betadinesoaked gauze also turned out to be less than successful in draining and cleansing the base of the wound. The physicians also considered making a drainage pathway from the deepest part of the wound all the way to the patient's backside, but they did not try it because this procedure is known to have high morbidity. The tube irrigation method was the procedure that was finally chosen. Though the wound shrank in size only very slowly, there were no further infections or aggravations. At 8 months since the flap coverage, there had been no complications.

A deep and narrow wound with a small opening permits little access to the wound bed and hinders mechanical cleansing. Wounds such as this typically have higher infection rates due lack of effective drainage and hygiene within the wound [5]. Physicians have previously tried various methods to treat deep and narrow wounds. Deschka et al. [1] have documented the use of continuous irrigation and drainage systems for spinal 


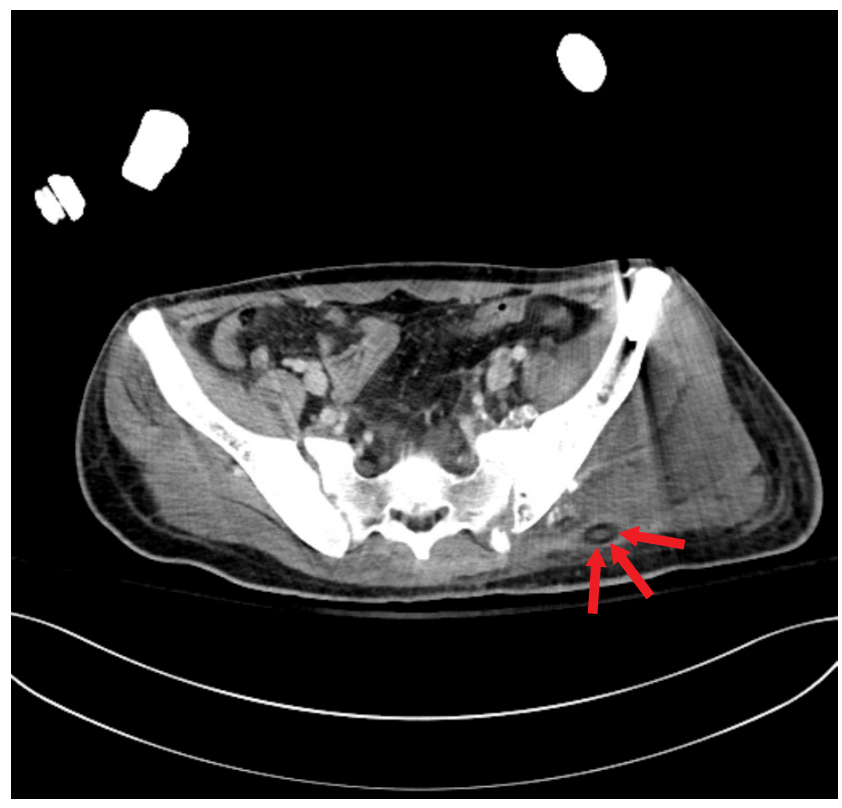

Fig. 6. CT scan showing the inserted irrigation tube. An irrigation tube (a rectal tube in this CT scan) is shown in this axial cut image (red arrows). Fluid collection can be seen just lateral to the rectal tube. $\mathrm{CT}$, computed tomography.

wounds. They managed patients with deep sternal wound infections after cardiac surgery with continuous suction-irrigation drainage and claimed successful outcomes [6]. Using pulsed lavage to cleanse a wound has also been reported by many authors $[7,8]$. Moreover, Yang and Dai [4] have presented the use of a side-hole double-valve lavage tube as an improvement on conventional lavage techniques.

The case described in this article, where the wound was irrigated using tubes, provided a number of new attempts. First, the course of the irrigation tube was tracked in imaging studies such as computed tomography (Fig. 6), ascertaining the irrigation was cleansing the necessary places throughout the narrow wound. Second, the color of the saline first irrigated provided insight on the nature of the pus or discharge from the deepest point of the wound. Furthermore, instead of using closed drain lines that only fit normal syringes with small openings, in this case rectal tubes or Foley catheters with wider openings that fit on enema syringes were used, making massive irrigation possible. The syringes also made it easy to control the irrigation pressure. Moreover, the universal tubes used in this case enabled use of other medical equipment, such as hanging a urine bag filled with saline on an intravenous stand and connecting it to the tube for continuous irrigation. Simply adjusting the height of the intravenous stand would change the rate of irrigation.
As for the shortcomings of the approach taken, first, the procedure failed to remove biofilms, potentially delaying the healing of the wound or making the wound chronic [9]. Second, there was an extended period of wound management before the flap coverage was performed, because the physicians had to wait for the pelvic bone to be stabilized. Further studies on multiple cases where this particular irrigation and management were applied over shorter durations would contribute to future wound care.

The steps taken in the case worked well for irrigating and cleansing, but did not contribute to draining the discharge. For better drainage, we suggest the use of NPWT devices with simultaneous instillation, as NPWT provides effective draining. Furthermore, the devices allow for continuous cleansing of the wound and control of the instillation rate. However, as the equipment diffuses the fluid to irrigate the wound, not even the most up-to-date machines can make the fluid target a specific point. In their investigation of the fluid dynamics of NPWT with simultaneous instillation, Davis et al. [10] found that though the instillation fluid spreads out rapidly in the demonstration model, the equipment fails to sufficiently cleanse the beds of deep and narrow wounds, as the bed rarely comes into contact with the NPWT sponge. Therefore, NPWT with instillation may not be suitable for cleansing the base of deep and narrow wounds. A combination of the irrigation demonstrated in the case of the 33-year-old male patient, and NPWT with instillation could be considered for managing deep and narrow wounds.

In conclusion, it is recommended to consider using tubes to irrigate deep and narrow wounds. Studies comparing different ways of cleansing and discussing new innovative methods, and also development and use of novel equipment including a combination of focal irrigation and NPWT are encouraged.

\section{Conflict of interest}

No potential conflicts of interest relevant to this article are reported.

\section{Acknowledgments}

Chan Min Chung https://orcid.org/0000-0002-2755-9440

Seung Wan Tak https://orcid.org/0000-0001-7744-5559

Hyoseob Lim https://orcid.org/0000-0002-6251-8281

Sang Hun Cho https://orcid.org/0000-0001-5906-9828 


\section{References}

1. Deschka H, Erler S, El-Ayoubi L, et al. Suction-irrigation drainage: an underestimated therapeutic option for surgical treatment of deep sternal wound infections. Interact Cardiovasc Thorac Surg 2013;17:85-9.

2. Born TR, Wagner ER, Kakar S. Comparison of open drainage versus closed catheter irrigation for treatment of suppurative flexor tenosynovitis. Hand (NY) 2017;12:579-84.

3. Yuan W, Liu X, Zhou X, et al. Management of early deep wound infection after thoracolumbar instrumentation: continuous irrigation suction system versus vacuum-assisted closure system. Spine (Phila Pa 1976) 2018;43:E1089-95.

4. Yang Z, Dai S. Treatment of infection with improved pulsed lavage. Wounds 2013;25:251-5.

5. Gerngross H, Engler V. Gravity drainage versus suction drain- age: an experimental and clinical study. Unfallchirurg 1989; 92:37-42.

6. Lian XF, Xu JG, Zeng BF, et al. Continuous irrigation and drainage for early postoperative deep wound infection after posterior instrumented spinal fusion. J Spinal Disord Tech 2014;27:E315-7.

7. Luedtke-Hoffmann KA, Schafer DS. Pulsed lavage in wound cleansing. Phys Ther 2000;80:292-300.

8. Muscatelli S, Howe A, O'Hara NN, et al. Comparison of irrigation times using gravity and high-pressure lavage. Orthopedics 2017;40:e413-6.

9. Rajpaul K. Biofilm in wound care. Br J Community Nurs 2015; Suppl Wound Care:S6-S10-1.

10. Davis KE, Moquin KJ, Lavery LA. The fluid dynamics of simultaneous irrigate with negative pressure wound therapy. Int Wound J 2016;13:469-74. 